\title{
Detecting compositional changes in dielectric materials simulated by three- dimensional RC network models
}

Article

Accepted Version

Galvão, R. K. H., Matsuura, J. P., Colombo Jr., J. R. and Hadjiloucas, S. (2017) Detecting compositional changes in dielectric materials simulated by three-dimensional $\mathrm{RC}$ network models. IEEE Transactions on Dielectrics and Electrical Insulation, 24 (2). pp. 1141-1152. ISSN 1070-9878 doi: https://doi.org/10.1109/TDEI.2017.006203 Available at https://centaur.reading.ac.uk/70166/

It is advisable to refer to the publisher's version if you intend to cite from the work. See Guidance on citing.

To link to this article DOI: http://dx.doi.org/10.1109/TDEl.2017.006203

Publisher: IEEE

All outputs in CentAUR are protected by Intellectual Property Rights law, including copyright law. Copyright and IPR is retained by the creators or other copyright holders. Terms and conditions for use of this material are defined in the End User Agreement. 


\section{CentAUR}

Central Archive at the University of Reading

Reading's research outputs online 


\title{
Detecting Compositional Changes in Dielectric Materials Simulated by Three-dimensional RC Network Models
}

\author{
Roberto K. H. Galvão, Jackson P. Matsuura, José Roberto Colombo Junior \\ Instituto Tecnológico de Aeronáutica, Divisão de Engenharia Eletrônica, \\ Praça Marechal Eduardo Gomes, 50, São José dos Campos, SP 12228-900, Brazil \\ and Sillas Hadjiloucas \\ School of Systems Engineering, \\ University of Reading, RG6 6AH, UK
}

\begin{abstract}
This work discusses the detection of small compositional changes in materials that have microstructures containing conducting and dielectric phases, which can be described by networks of resistive ( $R$ ) and capacitive (C) components in a three-dimensional lattice. For this purpose, a principal component analysis (PCA) method is employed to discriminate normal samples from samples with altered composition on the basis of statistics extracted from the waveform of the network response to a given excitation. This approach obviates the requirement for multivariate regression and simplifies experimental workload for model-building, since only data from normal samples are required in the development of the PCA model. Waveform variability of the excitation source is also accounted for through the use of a nominal model derived using subspace identification. This enables standardization and software based metrology transfer across different labs. The effect of network size on the capability of detecting minute compositional changes was assessed. For networks of 520 components, it was possible to identify changes in the fraction of capacitors down to $10^{-2}$ at $\pm 2 \sigma$ confidence levels.

Index Terms - Relaxation processes, Dielectric materials, Reliability modeling, Process control, compositional changes, screening analysis, PCA, RC networks.
\end{abstract}

\section{INTRODUCTION}

THERE are several complex phenomena that require the development of dielectric theories. Examples include interfacial and space charge movement at very low frequencies as well as orientational, ionic and electronic interactions associated with processes at progressively higher frequencies. In the 70's, Jonscher developed an empirical approach to the study of both conductors and insulators on the basis of their observed frequency-dependent permittivity according to a universal power law [1]. Subsequently, Lewis [2] as well as Dissado and Hill [3] suggested that a dielectric material may be visualized as a network of passive resistive and capacitive elements so that relaxation phenomena can be analyzed using equivalent circuits. Such postulations have significantly refined previous charge transport modelling approaches that were based on percolation and spectral density theories, collectively known as Archie's formulations or Kohlrausch-Williams-Watts models [4-6]. Following on the footsteps of the Hill and Dissado's approach, Almond's group [7-11], have systematically investigated the effect of changes in circuit parameters of randomly connected

Manuscript received on 31 July 2016, in final form 27 October 2016, accepted 30 November 2016. Corresponding author: S. Hadjiloucas.
RC networks studying their conductivity across a wide range of frequencies. Their work has placed electrical network theory at the core of current dielectric theories and has provided important theoretical evidence towards the validity of Lichtenecker's Mixture Formulae' which is based on the Effective Medium Theory $[12,13]$. Alternative statistical formulations confirming Jonscher's power law have also been proposed [14-18].

Following these formulations, appropriate networks can be derived and choice of circuit parameters can be made nowadays to reproduce the shape of the loss curve in ionic systems so as to provide further insights on charge transport on the basis of impedance spectroscopy results, taking also into consideration possible electron damping effects. A particularly useful application of these networks has been in predicting macroscopic conduction properties for composite materials [19-20]. Upon time domain excitation, these networks are shown to display a Warburg like impedance over a wide range of frequencies, matching the responses found in the dielectrics literature [19, 2132]. From a computational dielectrics perspective, it becomes therefore possible to systematically study small compositional changes of these materials by carefully studying changes in their emergent properties (which are otherwise difficult to identify because of an absence of clear resonance bands). To this end, 
large-scale RC network simulation models can be used to carry out computational investigations, modeling composite electrodes, assisting on the characterization of grain boundaries, providing alternative approaches and refining current deconvolution approaches as well as providing improved fidelity to variableslope models or composite models [31-33]. From an industrial applications perspective such simulations can also be particularly useful within a screening analysis context or from a quality control perspective.

A typical approach for screening analysis consists of using multivariate classification techniques to discriminate between "normal" and "faulty" samples on the basis of instrumental measurements. Such a discrimination is useful within the context of dielectrics as it provides a rapid method to check whether a given sample conforms to acceptance requirements of customers and/or regulatory agencies. Samples that do not pass the screening test may be subjected to more elaborate analysis procedures in order to confirm compliance or identify the reasons for nonconformity.

The present paper is concerned with the screening analysis of RC networks with respect to compositional changes on the basis of the time-domain response to an electrical input excitation. In previous work, we demonstrated the possibility of determining the fraction of capacitors in the network on the basis of time-domain or frequency-domain data [34], which were obtained from model based simulations [35]. However, such a procedure involved a multivariate regression procedure, which required the availability of calibration data from networks with different fractions of capacitors, which must be known a priori. Moreover, the effects of possible variabilities in the input excitation were not considered, i.e. it was implicitly assumed that the networks under test were all excited in exactly the same manner. In a related conference paper [36], we also demonstrated the possibility of detecting changes in the fraction of resistors in an RLC network by comparing the network output with the output of waveletbased models identified at various resolution levels. However, the proposed test protocol required the use of a wideband excitation input with reproducible spectral profile, which again may not be a realistic assumption for practical applications. In contrast, the screening method employed in the present work accounts for run-to-run variability in the excitation waveform and only requires the availability of data from networks with normal composition, which in practice simplifies the experimental workload for model-building. The adopted method is based on the test statistics commonly employed for detection of anomalies using Principal Component Analysis. In this context, the main contribution consists of introducing a model-based approach to account for possible variations in the excitation waveform.

\section{EFFECT OF COMPOSITIONAL CHANGES IN THE TIME-DOMAIN RESPONSE OF THREE-DIMENSIONAL RC NETWORKS}

Figure 1 illustrates the RC network topology considered in the present work. All the simulations were carried out by using networks with $N_{X}=5$ rows, $N_{Y}=5$ columns and $N_{Z}=3$ layers, with random allocation of the $R, C$ elements. As detailed in [35], the total number of resistors and capacitors in the network (excluding the source resistor $R_{S}$ ) is given by $N_{R C}=N_{Z}\left[N_{Y}\left(N_{X}+1\right)+N_{X}\left(N_{Y}-1\right)\right]+\left(N_{Z}-1\right) N_{X} N_{Y}$, which in this case amounts to $N_{R C}=200$. The resistance and capacitance values were normalized to $R=1, C=0.5$ and $R_{S}=$ 0.1 . Henceforth, the fraction of capacitors in the network will be denoted by $f_{C}$, with $f_{C}=0.5$ corresponding to the normal composition. A detailed description of the simulation model can be found elsewhere [35].

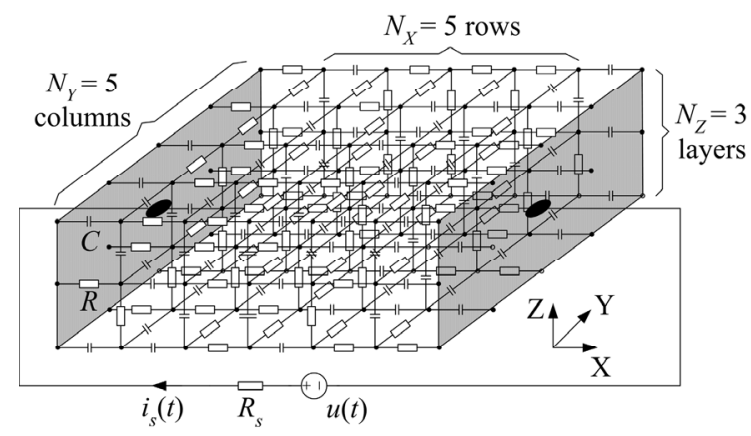

Figure 1. Network topology.

The input and output signals considered herein correspond to the voltage and current waveforms denoted by $u(t)$ and $i_{S}(t)$ in Figure 1. The simulations were initially carried out by using an input excitation signal of the form $u(t)=1-\exp (-t / \tau)$, with a time constant $\tau=0.1$ (normalized unit). Figure 2 shows the resulting current waveforms for a set of networks with normal $\left(f_{C}=0.5\right)$ and altered compositions $\left(f_{C}=0.4\right.$ in Figure $2 \mathrm{a}$ and $f_{C}=0.6$ in Figure 2b). Measurement imperfections were simulated by degrading the signals with white gaussian noise with zero mean and standard deviation of 0.01 (approximately $0.3 \%$ of the peak current value).

Despite the variability in the waveforms caused by the random allocation of the network elements, there is a noticeable difference between the responses obtained with normal (blue) and altered (red) compositions in Figures $2 \mathrm{a}$ and $2 \mathrm{~b}$. More specifically, by comparing the mean profiles presented in Figure $2 \mathrm{c}$, it can be seen that a reduction in $f_{C}$ is associated to smaller current values in the first half of the graph, and larger current values in the second half. Indeed, in a network with smaller $f_{C}$, the transient current is smaller because there are fewer capacitors being charged. In contrast, in a network with larger $f_{C}$, the steady-state current is smaller because the charged capacitors block the flow of current.

Additional insight into the differences between the current profiles can be gained by calculating a normalized difference index at each time $t$ as:

$$
I(t)=\frac{\mid \operatorname{mean}\left[i_{S, \text { altered }}(t)\right]-\text { mean }\left[i_{S, \text { normal }}(t)\right] \mid}{\operatorname{std}\left[i_{S, \text { normal }}(t)\right]}
$$

where mean $\left[i_{S, \text { altered }}(t)\right]$, mean $\left[i_{S, \text { normal }}(t)\right]$ denote the average current of the networks with normal and altered compositions, respectively, whereas std denotes the standard deviation. As 
can be seen in the inset of Figure $2 \mathrm{c}$, for $f_{C}=0.4$ the differences with respect to the normal case are more pronounced at the very beginning of the response. In contrast, for $f_{C}=0.6$, the largest normalized differences are found near the peak time.
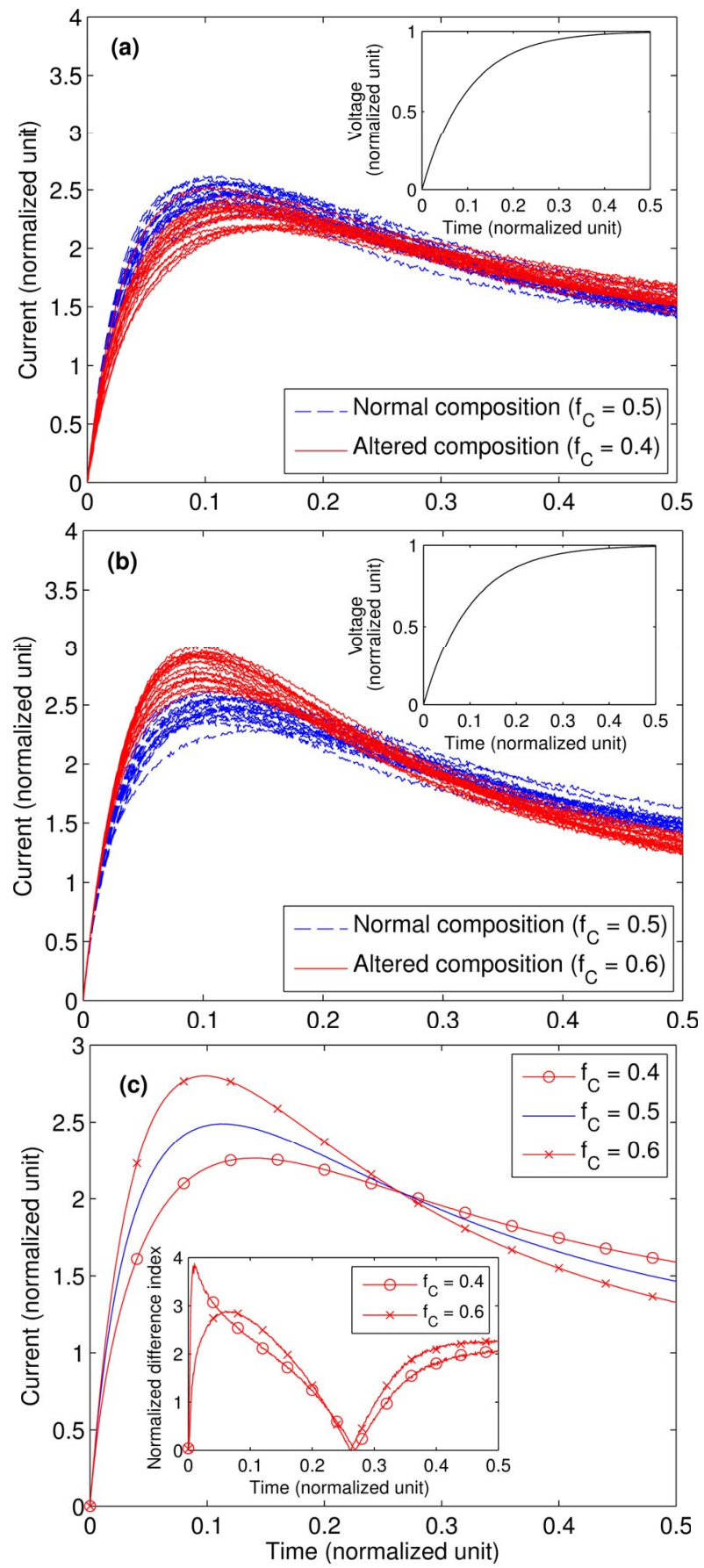

Figure 2. (a), (b) Responses of networks with normal and altered compositions to the input signal shown in the inset. (c) Mean profiles (inset shows normalized differences with respect to the normal case).

\section{INPUT WAVEFORM VARIABILITY}

In order to investigate the effect of variabilities in the input waveform, new simulations were carried out by using a different input signal to excite each of the networks with normal and altered compositions. These input signals were generated with random time constant values, distributed uniformly in the range from $\tau=0.05$ to $\tau=0.15$. As shown in Figures $3 \mathrm{a}$ and $3 \mathrm{~b}$, the discrimination between the responses of networks with normal (blue) and altered (red) composition is not so evident as in Figure 2a and 2b. Indeed, although the average current waveforms (Figure 3c) are similar to those presented in Figure 2c, the normalized differences (inset in Figure $3 \mathrm{c}$ ) are much smaller, due to the larger variability of the current values at each time $t$. In the case of $f_{C}=0.4$, the large initial peak of the normalized difference in the inset of Figure 2c was substantially reduced. This change can be attributed to the larger variability in the initial current values.
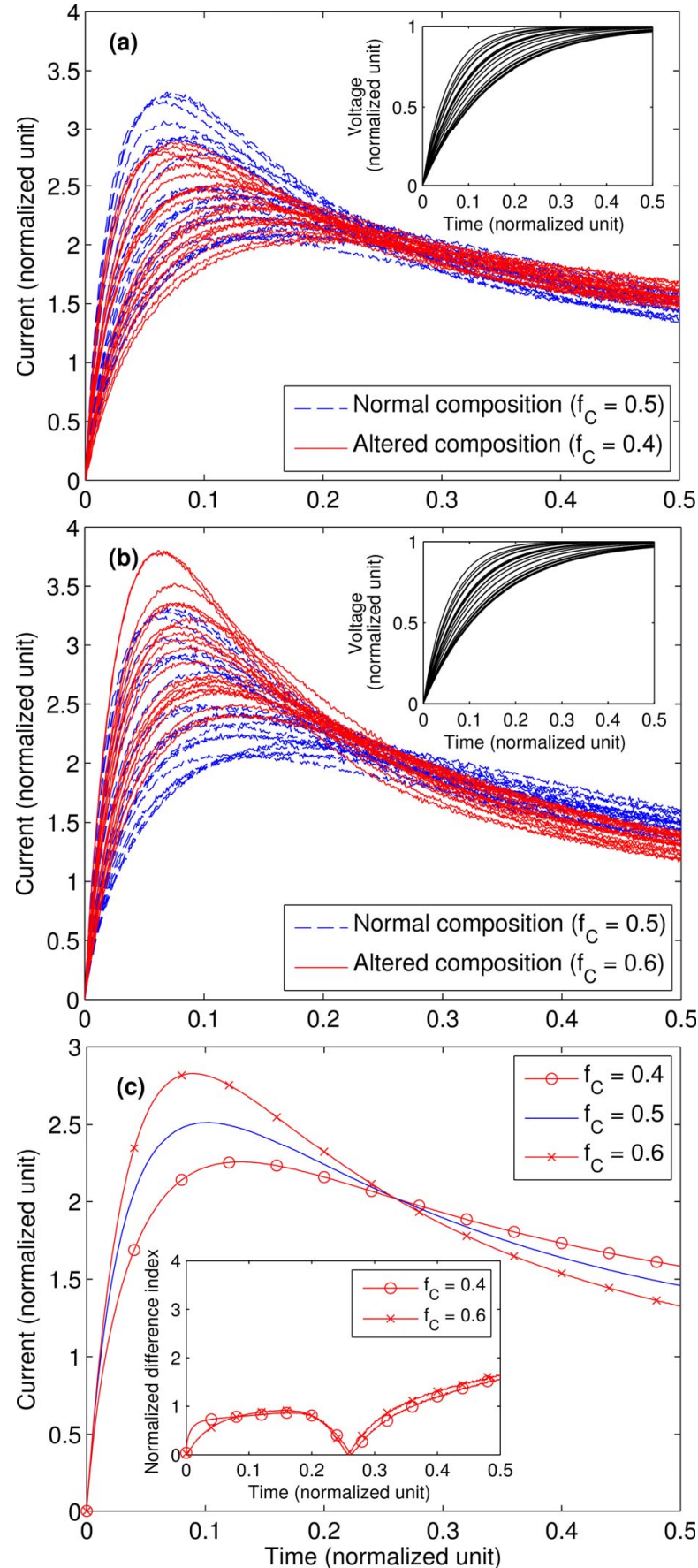

Figure 3. (a), (b) Responses of networks with normal and altered compositions in the presence of input variability (see inset). (c) Mean profiles (inset shows normalized differences with respect to the normal case). 


\section{MODEL IDENTIFICATION AND GENERATION OF RESIDUAL SIGNALS}

The method proposed herein to account for variations in the excitation waveform consists of obtaining a representative model for RC networks with normal composition and subsequently analyzing the difference between the model output and the response of the network under test. For this purpose, a discrete-time model for the dynamics of the network can be written in state-space form as:

$$
\begin{aligned}
& \mathbf{z}((k+1) T)=\mathbf{A z}(k T)+\mathbf{B} u(k T) \\
& i_{S}(k T)=\mathbf{C} \mathbf{x}(k T)+D u(k T)
\end{aligned}
$$

where $T$ is the sampling period, $k T$ denotes the $k^{\text {th }}$ sampling time and $\mathbf{z}(k T) \in \mathfrak{R}^{n}$ is a state vector. In the present work, the model matrices $\mathbf{A} \in \mathfrak{R}^{n \times n}, \mathbf{B} \in \mathfrak{R}^{n \times 1}, \mathbf{C} \in \mathfrak{R}^{1 \times n}, D \in \mathfrak{R}$ were obtained by using subspace identification [56] on the basis of voltage $u(t)$ and current $i_{S}(t)$ signals acquired by simulating a set of different networks with normal composition. This identification technique employs oblique projection operations involving the input-output data, followed by a singular value decomposition of the projection result. In the absence of noise, the number of nonzero singular values is equal to the true order $n$ of the system. In the presence of noise, an estimate for the system order can be obtained by retaining only the singular values that are significantly larger than zero [37].

The model thus obtained was employed to generate a residual signal $r(k T)$ as:

$$
\begin{aligned}
& \hat{\mathbf{z}}(0)=0 \\
& \hat{\mathbf{z}}((k+1) T)=\mathbf{A} \hat{\mathbf{z}}(k T)+\mathbf{B} u(k T) \\
& \hat{i}_{S}(k T)=\mathbf{C} \hat{\mathbf{z}}(k T)+D u(k T) \\
& r(k T)=i_{S}(k T)-\hat{i}_{S}(k T)
\end{aligned}
$$

where the hat symbol denotes quantities calculated by simulating the model for a given voltage waveform $u(k T)$. It is assumed that the networks are excited from a resting state, and thus $\hat{\mathbf{z}}(0)=0$.

\section{PCA SCREENING METHOD}

The screening method adopted in this work is based on Principal Component Analysis (PCA), using either the RC network response $i_{s}$ (electrical current waveform) or the residual signal $r$. It is assumed that a training set with data from $N$ networks of normal composition, acquired over $K$ sampling instants, is arranged in the form of a matrix $\mathbf{X}^{\text {train }}(N$ $\times K$ ). Each row of this matrix corresponds to an electrical current waveform or, in the case of the model-based approach, to the residual signal. It is assumed that the data have been mean-centered, as usual in PCA calculations.

PCA decomposes the matrix $\mathbf{X}^{\text {train }}$ in the form [38]

$$
\mathbf{X}^{\text {train }}=\mathbf{T} \mathbf{P}^{T}+\mathbf{E}
$$

where $\mathbf{T}(N \times R), \mathbf{P}(K \times R)$ and $\mathbf{E}(K \times R)$ are the matrices of scores, loadings and residues, respectively, for a given number of $R$ principal components.

The vectors of scores $\mathbf{t}^{\text {test }}(R \times 1)$ and residues $\mathbf{e}^{\text {test }}(K \times 1)$ for a new data vector $\mathbf{x}^{\text {test }}(K \times 1)$ are calculated as

$$
\begin{aligned}
\mathbf{t}^{\text {test }} & =\mathbf{P}^{T} \mathbf{x}^{\text {test }} \\
\mathbf{e}^{\text {test }} & =\mathbf{x}^{\text {test }}-\mathbf{P t}^{\text {test }}
\end{aligned}
$$

Two statistics are commonly used to check the consistency of the test data with the normal training set. The $D$ statistic, which corresponds to Hotelling's $\mathrm{T}^{2}$ metric within the reduced space of principal components, is calculated from the vector of scores $\mathbf{t}^{\text {test }}$ as $[38,39]$

$$
D=\left(\mathbf{t}^{\mathrm{test}}\right)^{T} \mathbf{S}_{\mathrm{T}}{ }^{-1} \mathbf{t}^{\mathrm{test}}
$$

where $\mathbf{S}_{\mathrm{T}}$ is the covariance matrix of the scores obtained from the training data, i.e.

$$
\mathbf{S}_{\mathrm{T}}=\frac{1}{N-1} \mathbf{T}^{T} \mathbf{T}
$$

It is worth noting that $\mathbf{S}_{\mathrm{T}}$ is diagonal due to the orthogonality of the scores [40]. Therefore, the $D$ statistic can also be expressed as [41]

$$
D=\sum_{i=1}^{R} \frac{\left(t_{i}^{\mathrm{test}}\right)^{2}}{s_{t_{i}}^{2}}
$$

where $t_{i}^{\text {test }}$ is the $i^{\text {th }}$ score of the test object and $s_{t_{i}}^{2}$ is the associated variance of $t_{i}$ in the training set.

The SPE (Squared Prediction Error) statistic is calculated from the vector of residues $\mathbf{e}^{\text {test }}$ as $[38,39]$

$$
S P E=\left(\mathbf{e}^{\text {test }}\right)^{T} \mathbf{e}^{\text {test }}
$$

The detection threshold $L_{D}$ for the $D$ statistic can be calculated as [39]:

$$
L_{D}=\frac{R\left(N^{2}-1\right)}{N(N-R)} F_{\alpha}(R, N-R)
$$

where $F_{\alpha}(R, N-R)$ is the critical value of an $F$ distribution with $R$ and $(N-R)$ degrees of freedom, at a given significance level $\alpha$.

As discussed in [38], a detection threshold $L_{S P E}$ for the $S P E$ statistic can be calculated, for a given significance level $\alpha$, by using an approximate distribution derived in [42].

Herein, an electrical response will be considered normal if and only if both statistics ( $D$ and SPE) are below the corresponding detection thresholds. The number of principal components $(R)$ employed in the analysis will be selected in order to explain $95 \%$ of the variance in the training data set.

It is worth noting that these statistics are often used in the context of multivariate statistical process control (MSPC). However, the following distinction should be pointed out. In typical MSPC applications, the $D$ and $S P E$ statistics are calculated from measurements of several sensors at each time, 
in order to detect process abnormalities. In the present work, these statistics are calculated for an entire waveform acquired from a single sensor, over a fixed time window.

It is also worth noting that the proposed use of a state-space model in Section 4 is not aimed at removing auto-correlations within the measured signal, as in standard MSPC applications [43], but rather at accounting for changes in the input excitation waveform.

\section{PERFORMANCE METRICS}

Following the usual terminology in screening analysis, the terms "negative" and "positive" will be used to indicate networks with normal and altered composition, respectively. A "false negative" will refer to a network with altered composition which passed the screening test (i.e. was not detected as being abnormal). In contrast, a "false positive" will refer to a network with normal composition which was incorrectly detected as having an altered composition (i.e., a false alarm).

The False Negative Rate $(F N R)$ and False Positive Rate $(F P R)$ obtained for a given test set with $n_{P}$ positive and $n_{N}$ negative objects are defined as

$$
F N R=\frac{F N}{n_{P}}, \quad F P R=\frac{F P}{n_{N}}
$$

where $F N$ and $F P$ are the number of false negatives and false positives, respectively. The Sensitivity (SEN) metric is calculated from the $F N R$ as $S E N=1-F N R$.

It is worth noting that the maximization of $S E N$ and the minimization of FPR are conflicting objectives. Indeed, by decreasing the detection threshold, a larger number of positive samples will be detected (i.e. SEN will get larger) at the cost of a larger number of false positives. The relation between these two metrics can be evaluated by varying the threshold and plotting a curve of $S E N$ versus $F P R$, which is often termed Receiver Operating Characteristic (ROC) curve [44]. $R O C$ curves are convenient to compare different detection methods, since the performance evaluation is not restricted to a fixed threshold value. More specifically, $R O C$ curves closer to the $S E N=1$ horizontal line are desirable, as they indicate a larger $S E N$ value for a given $F P R$. A metric usually employed in this comparison is the area under the ROC curve $(A U C)$. An $A U C$ closer to 1 indicates a better detection capability.

In what follows, all the performance metrics will be expressed in percent values. The results were obtained using the Matlab ${ }^{\circledR}$ 2012a software platform.

\section{RESULTS AND DISCUSSION}

The training set consisted of 500 networks with normal composition $\left(f_{C}=0.5\right)$. The screening analysis performance was evaluated by using test sets consisting of other 500 networks with normal composition $\left(f_{C}=0.5\right)$, as well as 500 networks of altered composition. Figure 4 presents the resulting $S P E$ vs $D$ plots for test sets in which the altered composition was set to $f_{C}=0.4$ (top plot) and $f_{C}=0.6$ (bottom plot). The normal test networks were the same in both cases. As can be seen, the networks with altered composition are associated to larger values of SPE and/or $D$. By setting the significance level to $\alpha=0.05, S E N$ values of $84.6 \%$ and $77.6 \%$ were obtained for the test networks with $f_{C}=0.4$ and $f_{C}$ $=0.6$ respectively. The $F P R$ value for the test networks with normal composition was $11.0 \%$.

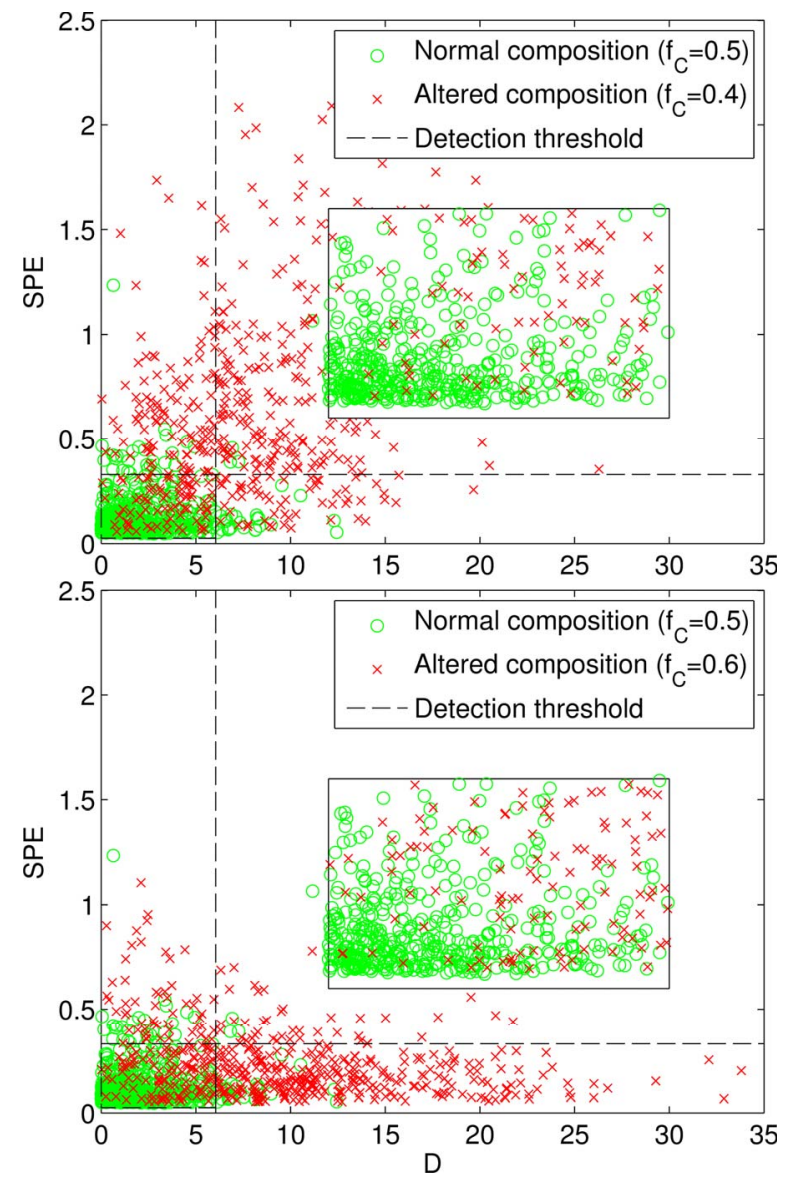

Figure 4. $S P E$ vs $D$ plots obtained by applying PCA to the network responses $(R=2 \mathrm{PCs})$. The vertical and horizontal dashed lines indicate the threshold values at a significance level $\alpha=0.05$. The insets present an enlarged view of the rectangle at the bottom left corner of each plot (region of normal objects).

Figure 5 present the SPE vS D plots obtained in the presence of variability in the input excitation waveform. As can be seen, the discrimination between networks with normal and altered composition becomes more difficult. Indeed, by keeping $\alpha=0.05$, the $F P R$ value for the test networks with normal composition was $9.2 \%$ (close to the value of $11.0 \%$ obtained in the absence of input variability), but the SEN values exhibited a substantial decrease to $28.6 \%$ and $41.0 \%$ for the test networks with $f_{C}=0.4$ and $f_{C}=0.6$ respectively. Interestingly, the reduction in $S E N$ was more pronounced for the networks with $f_{C}=0.4$, compared to the networks with $f_{C}=0.6$. Such a finding can be explained in terms of the larger impact of the input variability in the case $f_{C}=0.4$, as mentioned in the discussion of Figure 3.

In order to account for the input waveform variability, subspace identification was employed to obtain a nominal 
model for the networks with normal composition. A 2nd-order model was adopted, in agreement with the default settings of the computational package employed in the identification.

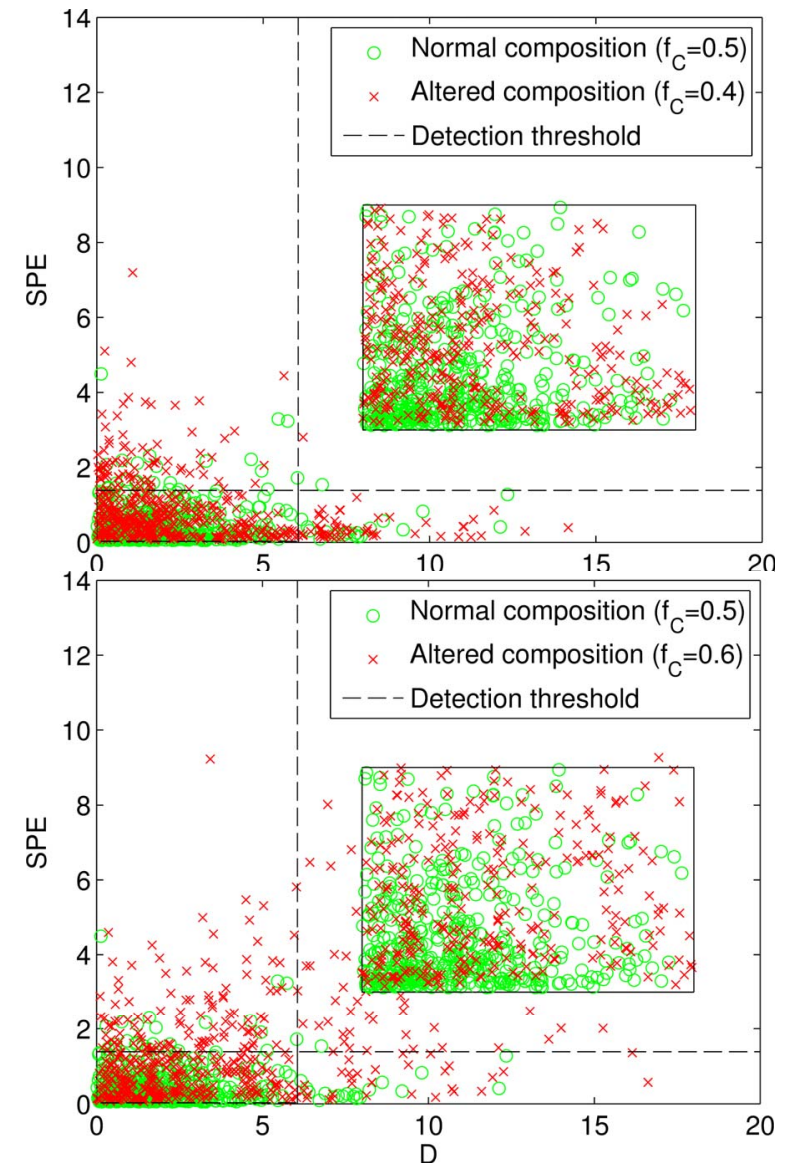

Figure 5. SPE vs D plots obtained by applying PCA to the network responses $(R=2 \mathrm{PCs})$ in the presence of input variability. The vertical and horizontal dashed lines indicate the threshold values at a significance level $\alpha=0.05$. The insets present an enlarged view of the rectangle at the bottom left corner of each plot (region of normal objects).

Figure 6 presents the residual signals obtained for the test networks. As can be seen, there is now a more clear distinction between the networks with normal (blue) and altered (red) composition, compared to Figure 3. It is worth noting that the residual signals for the test set of networks with normal composition (green) are similar to those of the training set.

The difference between the residual signals obtained for the networks with normal and altered composition is also apparent in Figure 7. This figure presents the mean profiles of the residual signals and the normalized differences (inset), which were calculated by using equation (1) with the residual signals instead of the current waveforms. By comparing these results with those presented in the inset of Figure 3c, it can be seen that the use of residual signals provided a substantial increase in the normalized difference index.

The better discrimination between the networks with normal and altered compositions is also apparent in the $S P E$ vs $D$ plots presented in Figure 8. Indeed, the $S E N$ values increased to $74.0 \%$ and $55.8 \%$ for the test networks with $f_{C}=$
0.4 and $f_{C}=0.6$ respectively, whereas the $F P R$ value remained at $10.4 \%$.

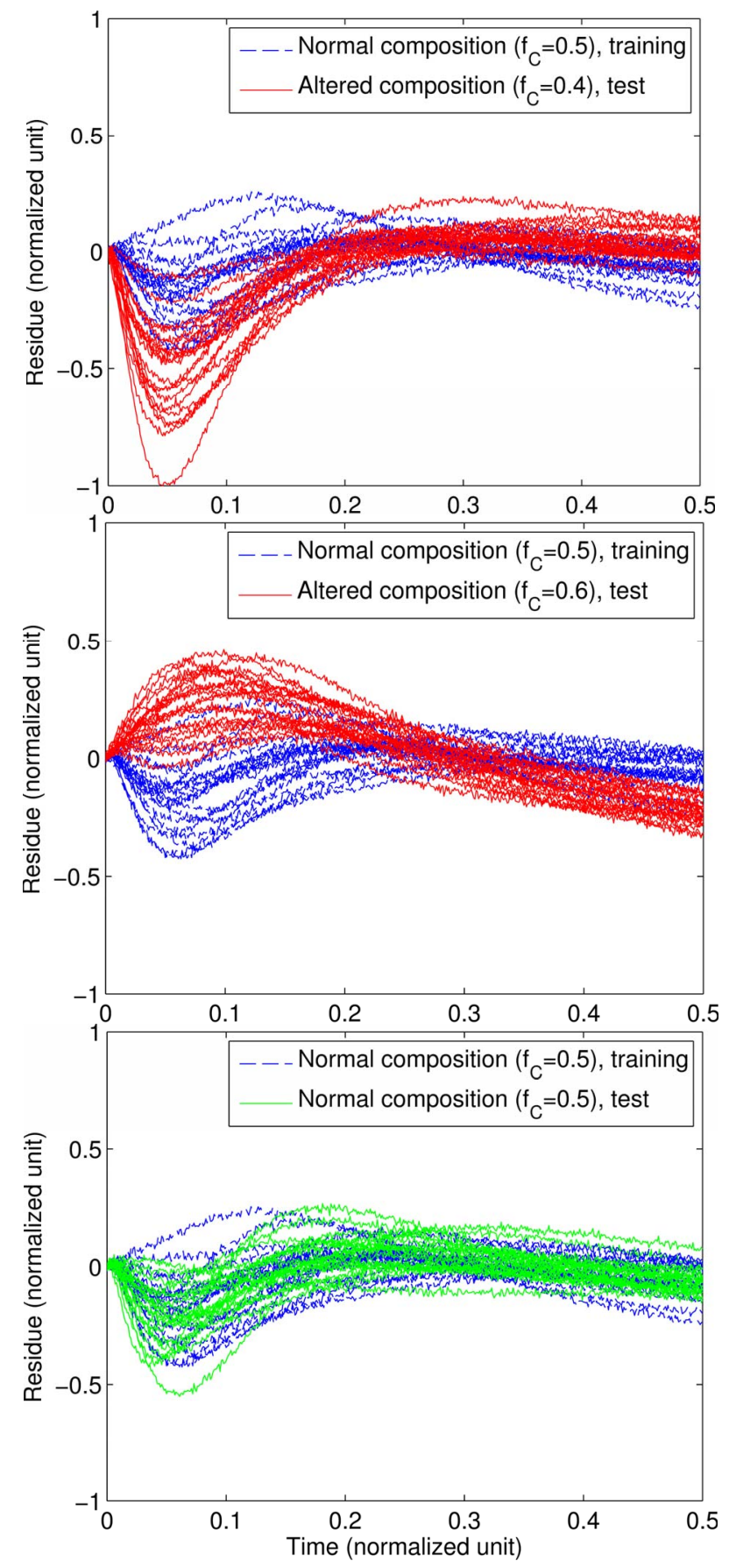

Figure 6. Residual signals obtained by using the identified model: Comparison between training and test sets.

The advantage of using the proposed model-based method to account for the input variability is also demonstrated by the $R O C$ curves presented in Figure 9. Such curves were obtained by calculating $S E N$ and FPR for different values of the significance level $\alpha$. By comparing the black and blue curves, it can be seen that the presence of variability in the input waveform degrades the detection capability, since SEN becomes smaller for any given FPR. However, this problem is mitigated by using the proposed model-based method (red 
curve), which provides an improvement in $S E N$ with respect to the sole use of the electrical current waveforms (blue curve).

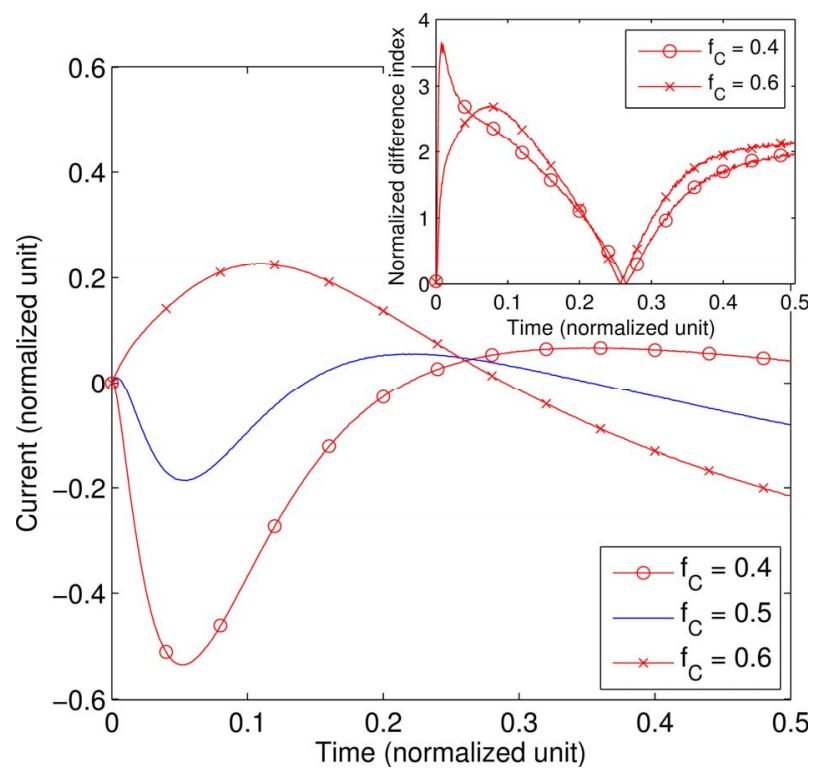

Figure 7. Mean profiles of the residual signals obtained by using the identified model (inset shows normalized difference with respect to the normal case).

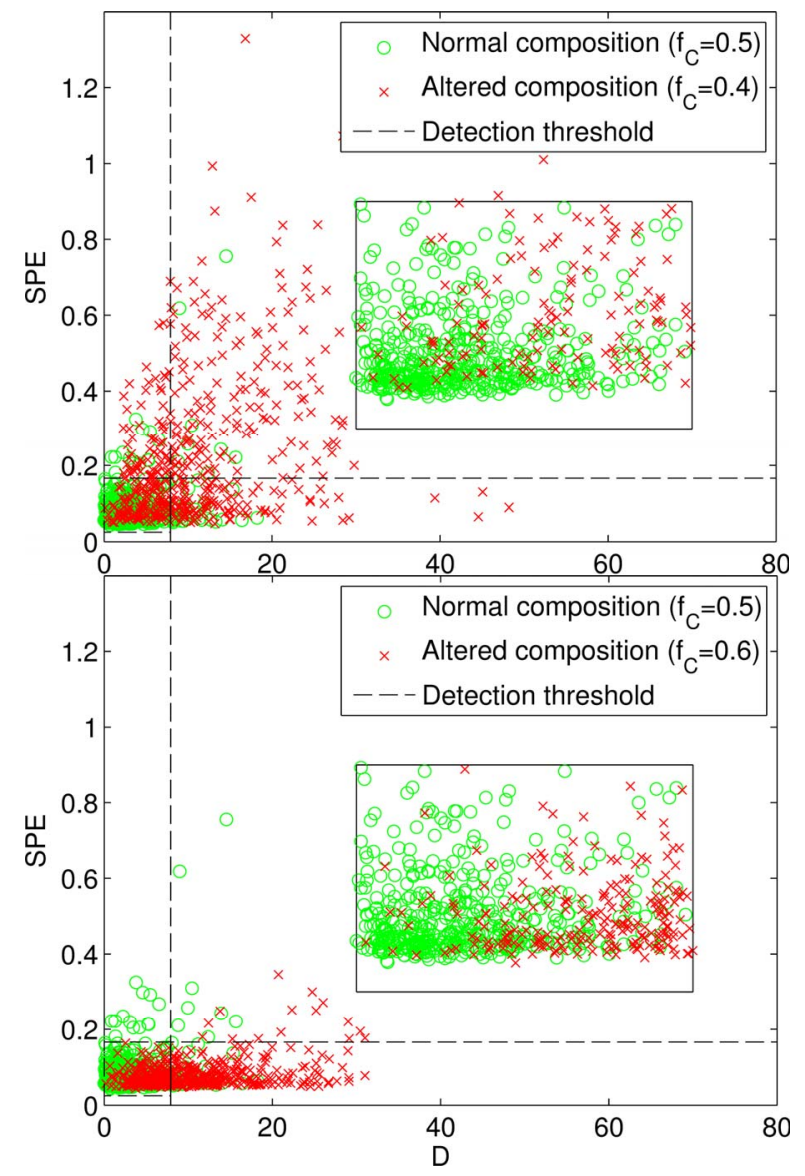

Figure 8. $S P E$ vs $D$ plots obtained by applying PCA to the residual signals $(R$ $=3 \mathrm{PCs})$ in the presence of input variability. The vertical and horizontal dashed lines indicate the threshold values at a significance level $\alpha=0.05$. The insets present an enlarged view of the rectangle at the bottom left corner of each plot (region of normal objects).
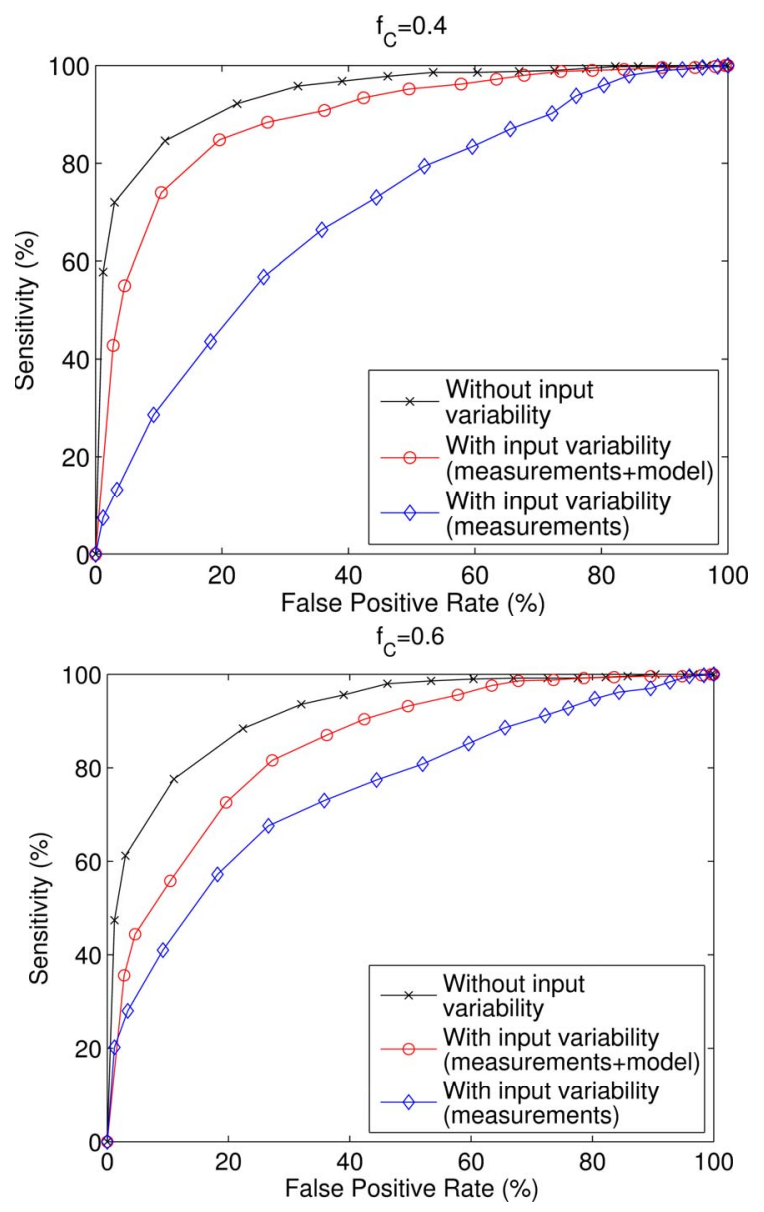

Figure 9. ROC curves obtained by varying the significance level $\alpha$.

Figure 10 presents the area under the $R O C$ curve $(A U C)$ for different values of the fraction of capacitors $f_{C}$ in the networks with altered composition. As expected, larger deviations of $f_{C}$ from the normal setting $\left(f_{C}=0.5\right)$ result in larger $A U C$ values, getting closer to the ideal value $(A U C=100 \%)$. The presence of input variability (blue curve) causes a reduction in $A U C$ compared to the case without input variability (black curve). This reduction is more prominent for smaller $f_{C}$ values, which is in agreement with the previous comments regarding Figure 3 and Figure 5. The proposed model-based method (red curve) provides a systematic improvement with respect to the sole use of the current waveforms (blue curve), leading to results that are closer to those obtained in the absence of input variability (black curve).

Finally, an investigation was carried out to assess the effect of the network size on the capability of detecting minute changes in its composition. As previously mentioned, the network depicted in Figure 1 has a total of 200 components. Smaller networks of 128 components $\left(N_{X}=4, N_{Y}=4, N_{Z}=3\right)$ and larger networks of 520 components $\left(N_{X}=8, N_{Y}=6, N_{Z}=\right.$ 4) were generated by varying the network dimensions while keeping the same ratio between the network length along the $X$ direction and its cross-section area.

Figure 11 presents a graph of the resulting AUC values for small changes of composition around the nominal value $f_{C}=$ 0.5 . For the networks of 128 and 200 components, the $f_{C}$ values 


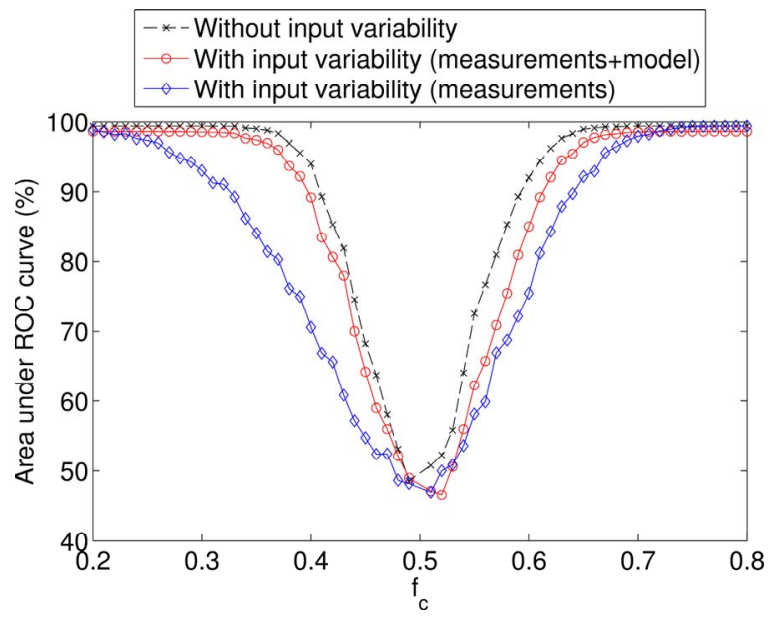

Figure 10. Area under the $R O C$ curve for different values of $f_{C}$.

were varied between 0.450 and 0.550 with 0.001 increments. For the networks of 520 components, the $f_{C}$ values were varied with an increment of $5 \times 10^{-4}$. The number of capacitors was always rounded to the nearest integer, which resulted in a quantization of the $f_{C}$ values. Each quantized $f_{C}$ was associated to a group of AUC values, with a corresponding mean and standard deviation $(\sigma)$. The error bars in Figure 11 indicate $\pm 2 \sigma$ intervals. It is worth noting that a detector with some ability to discriminate between samples with normal and altered composition must have an AUC larger than 50\% [45]. Therefore, the limits of detection (presented in the insets) were obtained as the smallest deviations from the nominal $f_{C}$ value beyond which the entire error bars were above $50 \%$.

The results in Figure 11 corroborate the previous discussion regarding Figure 10. Moreover, it can be seen that an increase in the network size facilitates the detection of compositional changes in the absence of input variability (black curves), as indicated by the reduction in the limits of detection. For networks with 520 components, changes of $\pm 10^{-2}$ in $f_{C}$ already result in AUC values significantly larger than $50 \%$. Indeed, the random allocation of $R, C$ elements has a smaller effect in the variability of the network response if the network size is increased. A detailed discussion can be found in [34]. In the presence of input variability, the proposed model-based method (red curves) enjoys a similar improvement in the detection capability as the network size increases. However, by using only the current waveforms (blue curves), the limit of detection for a decrease in $f_{C}$ (left-hand side of the graphs) is approximately the same for the three network sizes. This finding can be interpreted by noting that the input variability has a more detrimental effect on the detection capability when $f_{C}$ is smaller than the nominal value, as discussed with respect to Figures 3, 5 and 10.

\section{PLACING THE PROPOSED METHOD IN A BROADER CONTEXT TO DETECT COMPOSITIONAL CHANGES IN DIELECTRICS}

The proposed screening analysis methodology is well tailored to identify minute compositional changes in materials
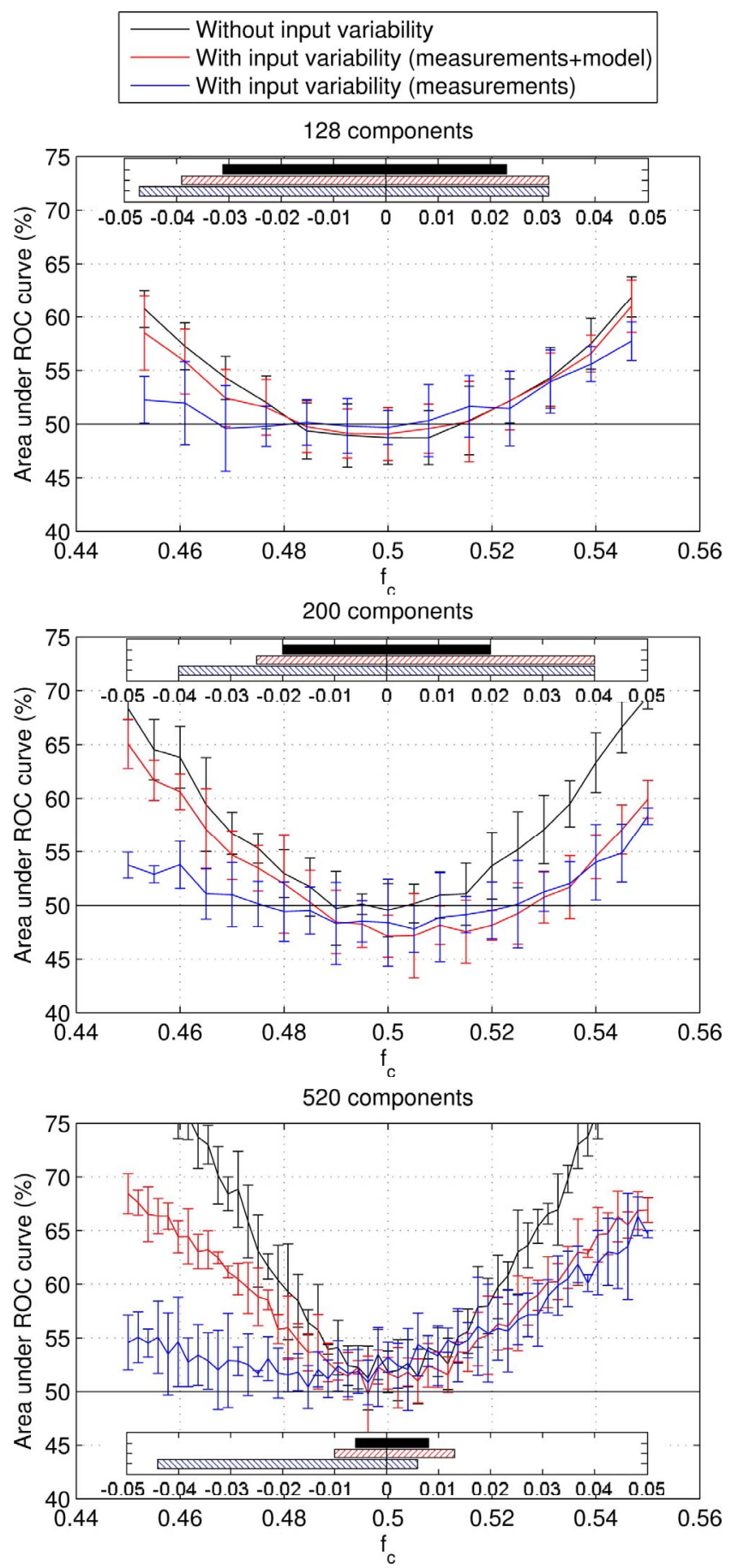

Figure 11. Area under the ROC curve for small deviations from the nominal composition. The error bars correspond to $\pm 2 \sigma$ intervals. The inset shows the smallest deviations from the normal composition beyond which the entire error bars are above $50 \%$.

that have microstructures containing conducting and dielectric phases, which can be described by RC networks such as those considered herein. In addition, the proposed statistical framework can provide new insights in complex diffusive charge transport processes in mesoscopic devices that display Warburg like impedances and enable the study of charge transport across a wide range of dielectrics and ferroelectrics [46-48]. From a technological perspective, there is scope to adopt the combined methodology of using $R C$ network models 
and screening analysis to reproduce and study possible alterations in the response of solid polymer electrolytes formed by the distribution of alkali metal salts (e.g. $\mathrm{LiClO}_{4}$ ) in polymers (e.g., PMMA, PAN, PEO, PVDF) which are recently finding new application in high-energy electrochemical devices due to their light weight, flexibility, ability to form intricate shapes, and low manufacturing cost [48-51].

The proposed methodology may also be combined with the use of other system identification tools [48], and should have applications across all impedance spectroscopy investigations, this can be particularly useful in emerging fields of physical chemistry such as synthetic metals [51] where Kanzaki forces and the gradient of the effective interaction potential can be related to the measured displacements in alloys [52].

Another particularly interesting application domain of combining screening analysis techniques with network models is in identifying minute compositional changes in samples displaying a photoinduced giant dielectric constant, as is found in lead halide perovskite solar cells [53]. Other application domains are in quality control for nanostructured ceramics [54] or the study of the effects of porosity, electrode and barrier materials on the conductivity of piezoelectric ceramics in high humidity and dc electric field [55] or generally to study the degradation of piezoelectric materials [56] as well as in supercapacitors research [57]. Moreover, compositional changes could be investigated in electrochemical studies [58-60] and dispersion chemistry, with applications in semiconductor surface states, colloidal semiconductors, and surface oxides [61-63].

The proposed methodology may also be applied to various spectroscopic investigations across the electromagnetic spectrum. Such approaches include datasets obtained using continuous wave Fourier transform spectrometers as well as time domain spectrometers using femtosecond lasers. Moreover, empirical modifications of the Debye relaxation such as the Cole-Cole equation, Cole-Davidson equation, the Havriliak-Negami relaxation and Kohlrausch-WilliamsWatts function (Fourier transform of stretched exponential function) are all compatible with the RC lattice responses assumed [64, 65]. Furthermore, applications can be found in microwave permittivity studies [66] or in studies of dielectric relaxation of salt solutions [67]. Previous work on the use of systems identification algorithms for the calculation of the complex insertion loss of a sample on the basis of acquired $\mathrm{THz}$ spectra from continuous wave or $\mathrm{THz}$ transient spectrometers [37, 68, 69] is also compatible with the proposed statistical framework.

Other recent studies where the proposed statistical models should prove particularly relevant to the dielectrics community include investigations of minute changes in composition associated with a change in the dielectric response of various partially cured epoxy nanocomposites [70], studies of charge transport and quality control in polyethylene nano-dielectrics [71], as well as relaxor terpolymer nanocomposites [72], performance in piezoelectric films [73], or quality control in enamel insulation [74] as well as other dielectric insulator materials as discussed in [75] and references therein.
The work also points towards the further development of a statistical framework to be used in deconvolution techniques for space charge recovery using pulsed electroacoustic methods [76] and other space charge measurements [77-79], in voltage-time characteristic curves [80] as well as electric modulus studies [81], and could be adopted in a wide range of studies, for example for the detection of changes in moisture content in the cellulosic part of power transformer insulation [82], studies of charge dynamics in mineral oils [83, 84], time domain dielectric relaxation studies on charging and discharging current of ferroelectric ceramics [85], nonlinear relaxational polarization [86], or could account for variation in the dielectric response of transformer oils [87]. Alternatively, it can account for statistical variation in the correlation between volume effect and lifetime relaxation times observed in experimental studies [88] or account for statistical variation in studies of ion mobilities in non-polar dielectric liquids [89] or when discussing the dielectric response of samples obtained using higher order spectroscopies [90]. Finally, another possible application domain is in providing an alternative way for understanding relaxation functions in glasses [91].

A further advantage of the proposed methodology is that it may be linked to the level of precision needed when measurements are made. In this case, a percentage of an acceptable false negative rate $F N R$ or false positive rate $F P R$ following a set of measurement will be associated with a signal to noise ratio or a precision of de-embedded lifetimes. This should directly benefit to the quality control community where often integration time in the measurement process may be limited or needs to be optimized. Furthermore, by explicitly accounting for input waveform variability, the work is also of relevance to digital data repositories, assisting the integration of experimental datasets from similar experiments performed in different laboratories. From a metrology perspective, the proposed statistical analysis enables an inter-comparison of measurements to be performed. In this case, the effect of certain amount of variability in the excitatory signal can be directly associated with the anticipated measurement precision.

An important further development of the proposed formulation is an extension to permit the inclusion of defects in the modeled three-dimensional RC structures (by changing the resistance or capacitance of components at specific locations in the network) to enable further investigations of the network response as a result of such defects [92]. This would be particularly useful in areas such as semiconductor detector technologies where donor or acceptor defects need to be introduced in a controlled manner as well as in superconducting materials where small changes in stoichiometry can have dramatic consequences in material properties.

\section{CONCLUSION}

A screening procedure where the $D$ and SPE statistics are calculated for an entire waveform over a fixed time window was successfully employed to detect compositional changes in RC networks simulating charge transport in dielectrics. This approach obviates the requirement for a multivariate 
regression procedure and further simplifies experimental workload for model-building, since only data from nominal networks are required. Moreover, a model-based procedure was proposed to account for waveform variability of the excitation source, this approach enables standardization and software based metrology transfer across different labs. The effect of network size on the capability of detecting minute changes in its composition was also assessed; for networks of 520 components, it was possible to identify changes in the fraction of capacitors down to $10^{-2}$ at $\pm 2 \sigma$ confidence levels. The proposed model-based screening methodology is generic and can be applied in a quality control context for the detection of compositional changes in datasets acquired using a very wide range of experimental modalities such as impedance, microwave, nuclear magnetic or electron spin resonance spectroscopy.

\section{ACKNOWLEDGMENT}

The authors acknowledge the support of FAPESP (grants 2011/17610-0 and 2011/13777-8), CNPq (research fellowship 303714/2014-0) and CAPES (PhD scholarship).

\section{REFERENCES}

[1] A. K. Jonscher, "Dielectric relaxation in solids. J. Phys. D Appl. Phys., Vol. 32, R57-R70, 1999.

[2] T. J. Lewis, "The dielectric behavior of non-crystalline solids," Dielectric and Related Molecular Processes, Vol. 3, pp. 186-218, 1977.

[3] L. A. Dissado and R. M. Hill, "Non-Exponential Decay in Dielectrics and Dynamics of Correlated Systems," Nature, Vol. 279, pp.685-689, 1979.

[4] M. Kaushik, B. W. H. Ng, B. M. Fischer and D. Abbott, "Terahertz scattering by granular composite materials: An effective medium theory," Appl. Phys. Lett., Vol. 100, No. 1, 011107, 2012.

[5] A. S. Nowick and B. S. Lim, "Electrical relaxations: Simple versus complex ionic systems," Phys. Rev. B, Vol. 63 184115, 2001.

[6] D. L. Sidebottom, P. F. Green and R. K. Brow, "Comparison of KWW and Power-Law Analyses of an Ion-Conducting Glass", J. Non-Cryst Solids, Vol. 183, pp. 151-160, 1995.

[7] W. Wieczorek, A. Zalewska, M. Siekierski and J. Przyluski, "Modelling the ac conductivity behaviour of composite polymeric electrolytes by the effective medium theory," Solid State Ionics, Vol. 86, pp.357-362 1996.

[8] D. P. Almond and C. R. Bowen, "Anomalous power law dispersions in ac conductivity and permittivity shown to be characteristics of microstructural electrical networks," Phys. Rev. Lett., Vol. 92, 157601, 2004.

[9] D.P. Almond, C.J. Budd, M.A. Freitag, G.W. Hunt, N.J. McCullen and N.D. Smith, "The origin of power-law emergent scaling in large binary networks," Phys.. A, Vol. 392, pp. 1004-1027, 2013.

[10] D.P. Almond and B. Vainas, "The dielectric properties of random R-C networks as an explanation of the universal power law dielectric response of solids," J. Phys. Condens. Matter, Vol. 11, pp. 9081-9093, 1999.

[11] R. Bouamrane and D. P. Almond, "The 'emergent scaling' phenomenon and the dielectric properties of random resistor-capacitor networks," J. Phys. Condens. Matter., Vol. 15, pp. 4089-4100, 2003.

[12] J. C. Dyre and T. B. Schrøder, "Universality of ac conduction in disordered solids," Rev. Mod. Phys., Vol. 72, pp. 873-892, 2000.

[13] T. Zakri, J. P. Laurent and M. Vauclin, "Theoretical evidence for Lichtenecker's mixture formulae' based on the effective medium theory," J. Phys. D: Appl. Phys., Vol. 31, pp. 1589-1594, 1998.

[14] A. Jurlewicz and K. Weron, "Relaxation of dynamically correlated clusters," J. Non-Cryst Solids, Vol. 305, pp. 112-121 2002.

[15] A. Stanislavsky, K. Weron and J. Trzmiel, "Subordination model of anomalous diffusion leading to the two-power-law relaxation responses," EPL (Europhysics Letters), Vol. 91, 40003, 2010.
[16] B. Szabat, K. Weron and P. Hetman, "Heavy-tail properties of relaxation time distributions underlying the Havriliak-Negami and the Kohlrausch-Williams-Watts relaxation patterns," J Non-Cryst Solids, Vol. 353, pp, 4601-4607, 2007.

[17] K. Weron, "A Probabilistic mechanism hidden behind the universal power law for dielectric relaxation: general relaxation equation," $J$. Phys. Condens. Matter, Vol. 3, pp. 9151-9162, 1991.

[18] K. Weron, "How to obtain the universal response law in the Jonscher screened hopping model for dielectric relaxation," J. Phys. Condens. Matter, Vol. 3, pp. 221-223, 1991.

[19] D. P. Almond, C. R. Bowen and D. A. S. Rees, "Composite dielectrics and conductors: simulation, characterization and design," J. Phys. D: Appl. Phys., Vol. 39, pp. 1295-1304, 2006.

[20] T. Jonckheere and J. M. Luck, "Dielectric resonances of binary random networks," J. Phys. A, Vol. 31, pp. 3687-3717, 1998.

[21] D. P. Almond, G. K. Duncan and A. R. West, "The determination of hopping rates and carrier concentrations in ionic conductors by a new analysis of ac conductivity," Solid State Ionics, Vol. 8, pp. 159-164, 1983.

[22] D. P. Almond, C. C. Hunter and A. R. West, "The extraction of ionic conductivities and hopping rates from ac conductivity data," J. Mater. Sci., Vol. 19, 3236-3248, 1984.

[23] D. P. Almond, B.Vainas and N. F. Uvarov, "A new analysis of the bulk ac electrical response of ionic conductors," Solid State Ionics, Vol. 111, pp. 253-261, 1998.

[24] D. P. Almond and A. R. West, "Impedance and modulus spectroscopy of "real" dispersive conductors," Solid State Ionics, Vol. 11, pp. 57-64, 1983.

[25] D. P. Almond and A. R. West, "Mobile ion concentrations in solid electrolytes from an analysis of a.c. conductivity," Solid State Ionics, Vol. 9, pp. 277-282, 1983.

[26] R. F. Hamou, J. R. Macdonald and E. Tuncer, "Dispersive dielectric and conductive effects in 2D resistor-capacitor networks," J. Phys. Condens. Matter, Vol. 21, No. 2, 025904, 2008.

[27] J. R. Macdonald, "Comparison of the universal dynamic response power-law fitting model for conducting systems with superior alternative models," Solid State Ionics, Vol. 133, pp. 79-97, 2000.

[28] J. R. Macdonald, "Surprising conductive and dielectric system dispersion differences and similarities for two Kohlrausch-related relaxation-time distributions," J. Phys. Condens. Matter, Vol. 18, pp. 629-644, 2005.

[29] N. J. Mccullen, D. P. Almond, C. J. Budd and G. W. Hunt, "The robustness of the emergent scaling property of random RC network models of complex materials," J. Phys. D: Appl. Phys., Vol. 42, 064001, 2009.

[30] E. Tuncer, "Structure-property relationship in dielectric mixtures: application of the spectral density theory," J. Phys. D: Appl. Phys., Vol. 38, pp. 223-234, 2005.

[31] E. Tuncer, "Distribution of relaxation times: An inverse problem," IEEE Trans. Dielectr. Electr. Insul., Vol. 19, pp. 1221-1225, 2012.

[32] B. Vainas, D. P. Almond, J. Luo and R. Stevens, "An evaluation of random RC networks for modelling the bulk ac electrical response of ionic conductors," Solid State Ionics, Vol. 126, pp. 65-80, 1999.

[33] A. Lasia, Modeling of impedance of porous electrodes," In Modeling and Numerical Simulations; Springer New York: 2009, pp 67-137.

[34] R. K. H. Galvão, K. H. Kienitz, S. Hadjiloucas, G. C. Walker, J. W. Bowen, S. F. C. Soares and M. C. U. Araújo, "Multivariate analysis of random three-dimensional $\mathrm{RC}$ networks in the time and frequency domains," IEEE Trans. Dielectr. Electr. Insul., Vol. 20, pp. 995-1008 2013.

[35] R. K. H. Galvão, S. Hadjiloucas, K. H. Kienitz, H. M. Paiva and R. J. M. Afonso, "Fractional order modeling of large three-dimensional RC networks," IEEE Trans. Circuits Syst. I, Regular Papers, Vol. 60, pp. 624-637, 2013.

[36] H. M. Paiva, M. A. Q. Duarte, R. K. H. Galvão and S. Hadjiloucas, "Wavelet based detection of changes in the composition of RLC networks," J. Phys.: Conf. Ser., Vol. 472, 012011, 2013.

[37] R. K. H. Galvão, S. Hadjiloucas, V. M. Becerra and J. W. Bowen, "Subspace system identification framework for the analysis of multimoded propagation of THz-transient signals," Meas. Sci. Technol., Vol. 16, pp. 1037-1053, 2005

[38] H.-J. Ramaker, E. N. M. van Sprang, J. A. Westerhuis and A. K. Smilde, "The effect of the size of the training set and number of principal components on the false alarm rate in statistical process monitoring," Chemometr. Intell. Lab., Vol. 73, pp. 181-187, 2004. 
[39] D. Aguado, A. Ferrer, J. Ferrer and A. Seco, "Multivariate SPC of a sequencing batch reactor for wastewater treatment," Chemometr. Intell. Lab., Vol. 85, pp. 82-93, 2007.

[40] P. Nomikos and J. F. MacGregor, "Multivariate SPC charts for monitoring batch processes," Technometrics, Vol. 37, pp. 41-59, 1995.

[41] T. Kourti and J. F. MacGregor, "Process analysis, monitoring and diagnosis, using multivariate projection methods," Chemometr. Intell. Lab., Vol. 28, pp. 3-21, 1995.

[42] J. E. Jackson and G. S. Mudholkar, "Control procedures for residuals associated with principal component analysis," Technometrics, Vol. 21, 341-349, 1979.

[43] S. W. Choi, J. Morris and I.-B. Lee, "Dynamic model-based batch process monitoring," Chem. Eng. Sci., Vol. 63, pp. 622-636, 2008.

[44] D. Ballabio and V. Consonni, , "Classification tools in chemistry. Part 1: linear models. PLS-DA," Anal. Methods, Vol. 5, pp. 3790-3798, 2013.

[45] S. H. Park, J. M. Goo and C.-H Jo, "Receiver operating characteristic (ROC) curve: Practical review for radiologists," Korean J. Radiology, Vol. 5, pp. 11-18, 2004.

[46] V. Stephanovich and E. Kirichenko, "The physical mechanisms of relaxation times distribution in inorganic and organic disordered ferroelectrics," J. Non-Cryst Solids, Vol. 353, pp. 4418-4421, 2007.

[47] N. Shukla, A. K. Thakur, A. Shukla and D. T. Marx, "Ion Conduction Mechanism in Solid Polymer Electrolyte: An Applicability of AlmondWest Formalism,”'Int'1. J. Electrochem. Sci., Vol. 9, pp.7644-7659, 2014.

[48] H. Schäfer, E. Sternin, R. Stannarius, M. Arndt and F. Kremer, "Novel approach to the analysis of broadband dielectric spectra," Phys. Rev. Lett., Vol. 76, pp. 2177-2180, 1996.

[49] M. Watanabe, K. Sanui, N. Ogata, T. Kobayashi and Z. Ohtaki, "Ionic conductivity and mobility in network polymers from poly (propylene oxide) containing lithium perchlorate," J Appl Phys, Vol. 57, pp. 123$128,1985$.

[50] C. M. Cooke, "Multimode Charge Transport in Electron Implanted PMMA," IEEE Trans. Dielectr. Electr. Insul. Vol. 22, No. 3 pp. 14341438, June 2015

[51] C. Chen, A. Kine, R. D. Nelson and J. C. LaRue, "Impedance spectroscopy study of conducting polymer blends of PEDOT: PSS and PVA," Synthetic Metals, Vol. 206, pp. 106-114, 2015.

[52] B. Chakraborty, "Static displacements and chemical correlations in alloys," EPL (Europhysics Letters), Vol. 30, pp. 531-536, 1995.

[53] D. P. Almond and C. R. Bowen, "An Explanation of the Photoinduced Giant Dielectric Constant of Lead Halide Perovskite Solar Cells," J. Phys Chem. Lett., Vol. 6, pp. 1736-1740, 2015.

[54] M. Cain and R. Morrell, "Nanostructured ceramics: a review of their potential," Appl. Organomet. Chem., Vol. 15, 321-330, 2001.

[55] P. Weaver, M. Cain, M. Stewart, A. Anson, J. Franks, I. Lipscomb, J. McBride, D. Zheng and J. Swingler, "The effects of porosity, electrode and barrier materials on the conductivity of piezoelectric ceramics in high humidity and dc electric field," Smart Mater. Struct., Vol. 21, 045012 (9pp), 2012.

[56] M. G. Cain, M. Stewart, M. Gee, "Degradation of piezoelectric materials," National Physical Laboratory, Teddington, Report CMMT (A) 148: p 7, 1999 .

[57] T. J. Freeborn, B. Maundy and A. S. Elwakil, "Measurement of supercapacitor fractional-order model parameters from voltage-excited step response," IEEE Trans. Emerg. Sel. Topics Circuits Syst., Vol. 3, pp. 367-376, 2013.

[58] M. E. Orazem, P. Agarwal, A. N. Jansen, P. T. Wojcik and L. H. GarciaRubio, "Development of physico-chemical models for electrochemical impedance spectroscopy," Electrochim. Acta, Vol. 38, pp. 190319111993.

[59] S. Hahne and B. Ploss, "Numerical Simulation of Frequency-dependent Nonlinear Interfacial Polarization in Electrolytes with Blocking Electrodes," IEEE Trans. Dielectr. Electr. Insul., Vol. 22, No. 3; pp. $1415-1418,2015$

[60] Z. Zhou, D. Zhang, J. He and M. Li, "Local Degradation Diagnosis for Cable Insulation based on Broadband Impedance Spectroscopy," IEEE Trans. Dielectr. Electr. Insul., Vol. 22, No. 4; pp. 2097-2107, Aug. 2015

[61] J. Hubbard and L. Onsager, "Dielectric dispersion and dielectric friction in electrolyte solutions. I,” J. Chem. Phys., Vol. 67, pp. 4850-4857, 1977.

[62] N. Nandi, K. Bhattacharyya and B. Bagchi, "Dielectric relaxation and solvation dynamics of water in complex chemical and biological systems," Chem. Rev., Vol. 100, pp. 2013-2046, 2000.
[63] G. Schwarz, "A theory of the low-frequency dielectric dispersion of colloidal particles in electrolyte solution," J. Phys. Chem., Vol. 66, pp. 2636-2642, 1962

[64] E. W. Montroll and J. T. Bendler, "On Lévy (or stable) distributions and the Williams-Watts model of dielectric relaxation," J. Stat. Phys., Vol. 34, pp. 129-162, 1984.

[65] B. Martin and H. Kliem, "Non-exponential Kohlrausch Polarization Currents in Solid Electrolytes," IEEE Trans. Dielectr. Electr. Insul., Vol. 22, No. 1, pp. 503-508, Feb. 2015

[66] M. J. Akhtar, H. B. Baskey, P. Ghising and N. M. Krishna, "Microwave Effective Permittivity of the Layered Dielectrics and Composites using the Nonlinear Mixing Model," IEEE Trans. Dielectr. Electr. Insul., Vol. 22, No. 3; pp. 1702-1710, June 2015.

[67] R. Buchner, G. T. Hefter and P. M. May, "Dielectric relaxation of aqueous $\mathrm{NaCl}$ solutions," J. Phys. Chem. A, Vol. 103, pp. 1-9, 1999.

[68] S. Hadjiloucas, R. K. H. Galvão, V. M. Becerra, J. W. Bowen, R. Martini, M. Brucherseifer, H. P. Pellemans, P. H. Bolívar, H. Kurz and J. M. Chamberlain, "Comparison of subspace and ARX models of a waveguide's terahertz transient response after optimal wavelet filtering," IEEE Trans. Microw. Theory Tech., Vol. 52, pp. 2409-2419, 2004.

[69] S. Hadjiloucas, R. K. H. Galvão, J. W. Bowen, R. Martini, M. Brucherseifer, H. P. Pellemans, P. H. Bolívar, H. Kurz, J. Digby and G. M. Parkhurst, "Measurement of propagation constant in waveguides with wideband coherent terahertz spectroscopy," J. Opt. Soc. Am. B, Vol. 20, pp. 391-401, 2003.

[70] I. Preda, J. Castellon, S. Agnel, H. Couderc, M. Fréchette, F. Gao, R. Nigmatullin, S.Thompson and A.-F. Vaessen, "Dielectric Response of Various Partially Cured Epoxy Nanocomposites," IEEE Trans. Dielectr. Electr. Insul., Vol. 20, No. 2 pp. 580-591, 2013,

[71] T. J. Lewis, "Charge Transport in Polyethylene Nano Dielectrics," IEEE Trans. Dielectr. Electr. Insul., Vol. 21, No. 2; pp. 497-502, April 2014.

[72] F. Bauer, L. Eyraud G. A. Samara and S. T. Choi, "Pressure-Induced Relaxor-to-Ferroelectric Crossover in Vinylidene Fluoride Relaxor Terpolymer: A Possible Explanation to the High Performance of the Terpolymer Nanocomposites," IEEE Trans. Dielectr. Electr. Insul., Vol. 22, No. 3, pp. 1455-1461, 2015

[73] P. Pondrom, J. Hillenbrand, G. M. Sessler, J. Bös and T. Melz, "Energy Harvesting with Single-layer and Stacked Piezoelectret Films," IEEE Trans. Dielectr. Electr. Insul., Vol. 22, No. 3; pp. 1470-1476, 2015.

[74] A. T. Hoang, Y. V. Serdyuk and S. M. Gubanski, "Mechanisms of Surface Potential Decay on Enamel Wire Coatings," IEEE Trans. Dielectr. Electr. Insul., Vol. 22, No. 6; pp. 3470-3480, December 2015.

[75] D. Das-Gupta and P. Scarpa, "Modeling of dielectric relaxation spectra of polymers in the condensed phase," IEEE Electr. Insul. Mag.., Vol. 15, No. 2, pp. 23-32, 1999.

[76] L. Galloy, L. Berquez, F. Baudoin and D. Payan, "PSpice Modeling of the Pulsed Electro-acoustic Signal," IEEE Trans. Dielectr. Electr. Insul., Vol. 21, No. 3 pp. 1143-1153, June 2014.

[77] G. Mazzanti, G. Chen, J. C. Fothergill, N. Hozumi, J. Li, M. Marzinotto, F. Mauseth, P. Morshuis, C. Reed, A. Tzimas and Kai Wu, "A Protocol for Space Charge Measurements in Full-size HVDC Extruded Cables," IEEE Trans. Dielectr. Electr. Insul., Vol. 22, No. 1; pp. 21-34, Feb. 2015.

[78] D. Min, W. Wang, and S. Li, "Numerical Analysis of Space Charge Accumulation and Conduction Properties in LDPE Nanodielectrics," IEEE Trans. Dielectr. Electr. Insul., Vol. 22, No. 3; pp. 1483-1491, 2015.

[79] Z. Lv, J. Cao, X. Wang, H. Wang, K. Wu and L. A. Dissado, "Mechanism of Space Charge Formation in Cross Linked Polyethylene (XLPE) under Temperature Gradient," IEEE Trans. Dielectr. Electr. Insul., Vol. 22, No. 6, pp. 3186-3196, 2015.

[80] G. Krithika and S. Usa, "v-t Characteristics using Extended Disruptive Effect Model for Impulses of Varying Front Times," IEEE Trans. Dielectr. Electr. Insul., Vol. 22, No. 4; pp. 2191-2195, 2015.

[81] F. Tian and Y. Ohki, "Electric Modulus Powerful Tool for Analyzing Dielectric Behavior,” IEEE Trans. Dielectr. Electr. Insul., Vol. 21, No. 3; pp. 929-931, 2014.

[82] A. Baral and S. Chakravorti, "Prediction of Moisture Present in Cellulosic Part of Power Transformer Insulation using Transfer Function of Modified Debye Model," IEEE Trans. Dielectr. Electr. Insul., Vol. 21, No. 3, pp. 1368-1375, 2014.

[83] Y. Zhou, M. Hao, G. Chen, G. Wilson and P. Jarman, "Quantitative Study of Electric Conduction in Mineral Oil by Time Domain and Frequency Domain Measurement," IEEE Trans. Dielectr. Electr. Insul., Vol. 22, No. 5; pp. 2601-2610, 2015. 
[84] J. Gao, L. Yang, Y. Wang, C. Qi, J. Hao and J. Liu, "Quantitative Evaluation of Ageing Condition of Oil-paper Insulation Using Frequency Domain Characteristic Extracted from Modified Cole-Cole Model," IEEE Trans. Dielectr. Electr. Insul., Vol. 22, No. 5; pp. 26942702, October 2015.

[85] L. Zhao and X. Wei, "Time Domain Dielectric Relaxation Study on Charging and Discharging Current of Barium Stannate Titanate Ferroelectric Ceramics," IEEE Trans. Dielectr. Electr. Insul., Vol. 22, No. 2, pp. 728-733, 2015.

[86] L. Kankate, A. Gratsov and H. Kliem, "Nonlinear Relaxational Polarization in Aluminum Oxide," IEEE Trans. Dielectr. Electr. Insul., Vol. 22, No. 2, pp. 1220-1231, 2015 .

[87] K. Bandara, C. Ekanayake and T. K. Saha "Modelling the Dielectric Response Measurements of Transformer oil," IEEE Trans. Dielectr. Electr. Insul., Vol. 22, No. 2; pp. 1283-1291, 2015.

[88] L. Zhao., J. Su., Y. Pan., R. Li, B. Zeng, J. Cheng, and B. X. Yu, "Correlation between Volume Effect and Lifetime Effect of Solid Dielectrics on Nanosecond Time Scale," IEEE Trans. Dielectr. Electr. Insul., Vol. 22, No. 4, pp. 1769-1776, 2015.

[89] W. F. Schmidt and K. Yoshino, "Ion Mobilities in Non-Polar Dielectric Liquids: Silicone Oils," IEEE Trans. Dielectr. Electr. Insul., Vol. 22, No. 5, pp. 2424-2427, 2015.

[90] R. Olmi and M. Bittelli, "Dielectric Data Analysis: Recovering Hidden Relaxations by Fourth-order Derivative Spectroscopy," IEEE Trans. Dielectr. Electr. Insul. Vol. 22, No. 6; pp. 3334-3340, 2015.

[91] R. Hilfer, "Analytical representations for relaxation functions of glasses," J. Non-Cryst Solids, Vol. 305, pp. 122-126, 2002.

[92] H. Kanzaki, "Point defects in face-centred cubic lattice-II X-ray scattering effects," J. Phys. Chem. Solids, Vol. 2, pp. 107-114, 1957.

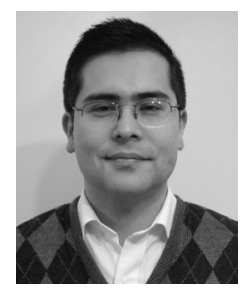

Roberto Kawakami Harrop Galvão (S'96-A'99M'03-SM'05) received the B.Sc. degree in electronic engineering (with Summa cum Laude honours) and the M.Sc. and Ph.D. degrees in systems and control from Instituto Tecnológico de Aeronáutica (ITA), Brazil, in 1995, 1997 and 1999, respectively. Since 1998 he has been with the faculty of the Electronic Engineering Department of ITA, where he is currently an Associate Professor of Systems and Control. In 2001 he spent a sabbatical leave at the Cybernetics Department of the University of Reading, UK. Dr. Galvão is a recipient of the Montenegro Award for Teaching Excellence at ITA. His research interests include fault diagnosis and prognosis, model predictive control and chemometrics.

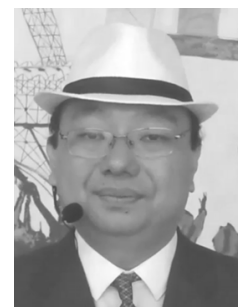

Jackson Paul Matsuura received the B.Sc. degree in computer engineering and the M.Sc. and Ph.D. degrees in systems and control from Instituto Tecnológico de Aeronáutica (ITA), Brazil, in 1995, 2003 and 2006, respectively. In 2009, he spent a post-doctoral period at the Institute of Systems and Robotics - University of Coimbra. Since 2005 he has been with the faculty of the Electronic Engineering Department of ITA, where he is currently an Adjunct Professor of Systems and Control. His research interests include fault diagnosis and prognosis, and robotics.

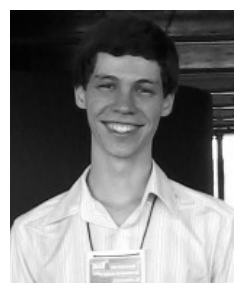

José Roberto Colombo Junior received the B.Sc. degree in electrical engineering from Universidade Estadual Paulista Júlio de Mesquita Filho (UNESP), Brazil, in 2013 and the M.Sc. degree in systems and control from Instituto Tecnológico de Aeronáutica (ITA), Brazil, in 2015, respectively. He is currently a $\mathrm{PhD}$ candidate at the Systems and Control group of ITA. His research interests include system identification and model predictive control.

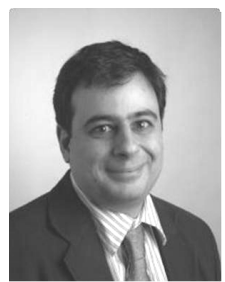

Sillas Hadjiloucas (M'94-SM'13) received the B.Sc. (Hons.) M.Phil. degrees in pure and applied biology from the University of Leeds, UK, in 1989 and 1992, respectively and in 1996, a Ph.D. degree in cybernetics from the University of Reading UK. After a short career break at the Greek army in 1997, and two appointments as an EC TMR Post-Doctoral Research Fellow working on $\mathrm{THz}$ Instrumentation as part of the INTERACT project in UK and the Deutsches Zentrum für Luft- und Raumfahrt (DLR Berlin), in January 2000 he was appointed Lecturer in Systems Engineering, at Reading university. His expertise is in instrumentation and measurement across the Optical, IR, THz and microwave parts of the spectrum, the studying of ultrafast phenomena using femtosecond lasers and the application of control theory and system identification techniques to spectrometry and systems biology. He is a reviewer for more than 50 different journals and AE for IEEE Sensors. He currently serves as Chair of the IoP Instrument Science and Technology Group and is a Fellow of the Institute of Measurement and Control. He is a Committee member of the Dielectrics Group of the IoP and chaired the 2013 IoP Dielectrics Conference at Reading. 